\title{
Competing Periodicities in Fractionally Filled One-Dimensional Bands
}

\author{
P. C. Snijders, ${ }^{1}$ S. Rogge, ${ }^{1}$ and H. H. Weitering ${ }^{2}$ \\ ${ }^{1}$ Kavli Institute of NanoScience, Delft University of Technology, 2628 CJ Delft, The Netherlands \\ ${ }^{2}$ Department of Physics and Astronomy, The University of Tennessee, Knoxville, Tennessee 37996, USA, \\ and Condensed Matter Sciences Division, Oak Ridge National Laboratory, Oak Ridge, Tennessee 37831, USA \\ (Received 18 October 2005; published 23 February 2006)
}

\begin{abstract}
We present a variable temperature scanning tunneling microscopy and spectroscopy study of the $\mathrm{Si}(553)-\mathrm{Au}$ atomic chain reconstruction. This quasi-one-dimensional system undergoes at least two charge density wave (CDW) transitions, which can be attributed to electronic instabilities in the fractionally filled $1 \mathrm{D}$ bands of the high-symmetry phase. Upon cooling, $\mathrm{Si}(553)$-Au first undergoes a single-band Peierls distortion, resulting in period doubling along the chains. This Peierls state is ultimately overcome by a competing $\times 3 \mathrm{CDW}$, which is accompanied by a $\times 2$ periodicity in between the chains. These locked-in periodicities indicate small charge transfer between the nearly $1 / 2$-filled and $1 / 4$-filled bands. The presence and the mobility of atomic-scale dislocations in the $\times 3 \mathrm{CDW}$ state indicates the possibility of manipulating phase solitons carrying a (spin, charge) of $(1 / 2, \pm e / 3)$ or $(0, \pm 2 e / 3)$.
\end{abstract}

According to the Mermin-Wagner theorem [1], thermodynamic fluctuations preclude the formation of a longrange ordered broken symmetry state in one dimension, except at $T=0 \mathrm{~K}$ [2]. For all practical purposes, however, thermodynamic phase transitions may still be possible in finite size 1D systems. Furthermore, fluctuations are inevitably suppressed if the 1D chains are weakly coupled, or if the chains are coupled to a substrate [2,3]. Prototypical 1D metallic systems such as the transition metal trichalcogenides, organic charge transfer salts, blue bronzes, and probably all atomic Au-chain reconstructions on vicinal Si substrates exhibit symmetry breaking phase transitions at finite temperatures $[4,5]$. For a band filling of $1 / n$, the phase transition opens up a gap in the single particle excitation spectrum at wave vector $k_{F}=\pi / n a$, and the corresponding broken symmetry state adopts the new periodicity of $\lambda=\pi / k_{F}=n a$, where $a$ is the lattice parameter of the high-symmetry phase [4].

Fractional band fillings other than half filling provide an interesting subset of 1D systems which often exhibit exotic physical phenomena. Depending on the relative magnitude of bandwidth and electron-electron interaction, charge density wave (CDW) states often compete with spin density waves, Mott insulating states, or a Luttinger liquid state. Atomic-scale STM observations of surface phase transitions provide important insights into the complexity of symmetry breaking phenomena in reduced dimensionality [6]. For instance, the recently reported $4 \times 1$-to- $8 \times 2$ phase transition in quasi-1D indium chains on $\mathrm{Si}(111)$ [6] involves a gap opening in a complex triple band Peierls system, resulting in a doubling of the periodicity along the atom chains. Another recently discovered system with three fractionally filled bands is the $\mathrm{Si}(553)$-Au surface. Angle-resolved photoemission spectroscopy (ARPES) [7] revealed three metallic bands, but despite theoretical efforts to understand the electronic structure $[7,8]$, the atomic structure and real space location of the surface state orbitals remains unknown. Interestingly, the total band filling of this particular chain system is $4 / 3$, i.e., corresponding to $8 / 3$ electrons per surface unit cell. Two bands have a filling of 0.56 and 0.51 each, slightly more than half filling. The third band has a filling of 0.27 , in between one-quarter and one-third filling.

In this Letter we present evidence for a defect mediated CDW transition [9] in $\mathrm{Si}(553)-\mathrm{Au}$ accompanied by a metal-insulator transition. STM experiments reveal competing periodicities as a function of temperature which can be mapped onto the band structure of the high-symmetry phase. Scanning tunneling spectroscopy (STS) data show a gradual gap opening at low temperatures, which can be correlated with successive gap openings in the three 1D bands. Interestingly, phase slips are observed in the CDW condensate. These phase slips should possess a fractional charge and a half integer or integer spin. The chain length can be tuned by manipulating the numerous defects with the STM tip. This, in turn, suggests the feasibility of studying and manipulating fractional charges at the atomic scale.

The $\mathrm{Si}(553)-\mathrm{Au}$ structure was prepared by depositing 0.24 monolayer of Au at a rate of 0.005 monolayer/s with the substrate held at $920 \mathrm{~K}$, followed by thermal annealing at $1120 \mathrm{~K}$ for $1 \mathrm{~min}$ and slow cooling to room temperature $\left(1 \mathrm{~K} \mathrm{~s}^{-1}\right)$. STM and STS experiments were performed in an omicron variable temperature STM. All distances determined in STM images were measured along the fast scan direction of the STM so as to minimize possible effects of thermal drift.

Figure 1 shows an STM image of the surface taken at room temperature (RT). Rather wide chains $(\sim 0.8 \mathrm{~nm})$ with a spacing of $1.48 \mathrm{~nm}$ are observed. The chains are cut by point defects appearing as vacancies in both filled state and empty state images. In the empty state image, 


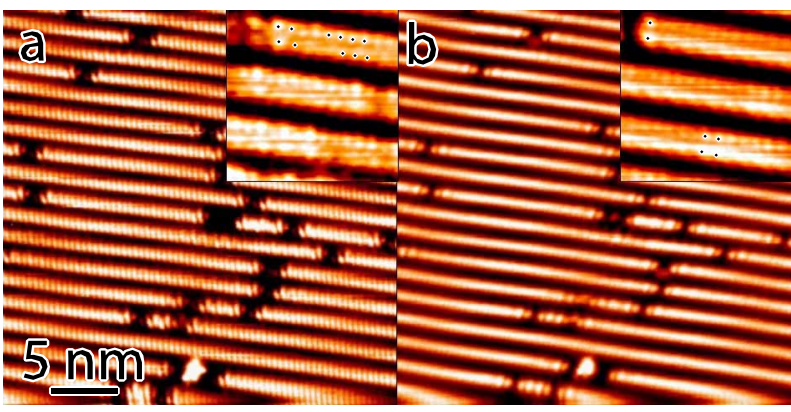

FIG. 1 (color online). (a) Empty state and (b) filled state $( \pm 0.5 \mathrm{~V}, 50 \mathrm{pA})$ STM images taken simultaneously at RT. Insets show magnifications. The structure in the chains is indicated with dots.

these vacancies appear larger than in the filled state image, confirming the observations reported in Ref. [10]. Note, however, that fewer defects are present as compared to other works $[7,10]$, indicating that the defect concentration can possibly be varied by controlling the annealing history of the surface despite propositions that the defects might be intrinsic to the surface as stabilizing charge dopants [7]. The insets show high resolution dual bias images. Clearly, the chains are composed of two rows of atoms, showing a zigzag structure in the empty state and a ladder configuration in the filled state. In the empty state image, all atom spacings are nearly equal to the bulk spacing of $\mathrm{Si}, a \sim$ $3.8 \AA$, resulting in bond angles near $60^{\circ}$. In the filled state image the spacing along the chain is equal to $a$, whereas the spacing perpendicular to the chains is $\sim 4.7 \AA$. These data seem to be inconsistent with structure models that place $\mathrm{Si}$ honeycomb chains [11] near the step edges [7,8] and with the single atom row structure suggested in Ref. [10].

Upon cooling to $\sim 40 \mathrm{~K}$, the zigzag features are no longer observed. Instead, an up-down buckling with a ladder structure in a tripled unit cell is observed (Fig. 2), resulting in two features of about $1.5 a$ length and widths comparable to those observed at RT. The corrugation of the filled state image is in antiphase with that of the empty state image; at the location of the intensity maxima in the

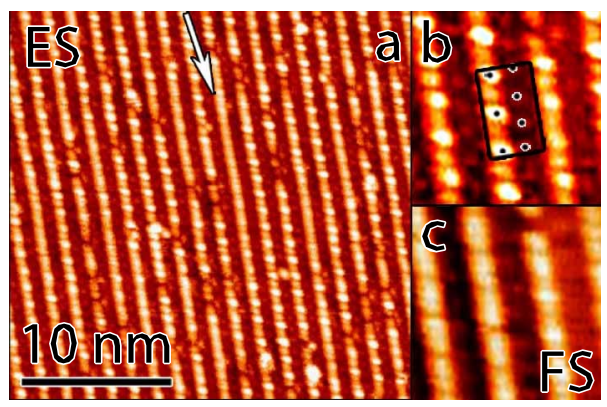

FIG. 2 (color online). (a) Empty state (1 V, 100 pA) STM image at $40 \mathrm{~K}$ and magnification (b). In (b) a $6 \times 1$ unit cell is indicated with combined $\times 3$ and $\times 2$ features. (c) Filled state $(-1 \mathrm{~V}, 100 \mathrm{pA})$ STM image corresponding to (b). empty state image, a small dip exists in the filled state image. This indicates that the system has condensed into a CDW with tripled periodicity, commensurate with the substrate lattice. Additionally, the features in the valleys between the chains visible in the empty state are completely ordered with a doubled period of $2 a$, resulting in a unit cell of $6 \times 1$.

The $I-V$ curves and their numerical derivatives are shown in Fig. 3. The $I-V$ curve at RT exhibits a significant slope at zero bias, confirming the metallicity observed in ARPES experiments [7]. In contrast, at $40 \mathrm{~K}$ the $I-V$ curve is flat at zero bias indicating semiconducting behavior. We determine the size of the gap from the derivative of the $I-V$ curves as displayed in the inset of Fig. 3; we infer that the excitation gap is symmetric and $\sim 150 \mathrm{meV}$ wide. Thus, the condensation of this CDW is accompanied by a metalinsulator transition. Although CDW instabilities in related surface systems are often manifested by an order-disorder transition [12], the present observations are most straightforwardly interpreted in terms of a displacive CDW transition.

The condensation scenario involving all three 1D bands is elucidated by STM experiments at temperatures intermediate between RT and $40 \mathrm{~K}$. Figure 4 shows empty state images measured at 70 and $110 \mathrm{~K}$. At $70 \mathrm{~K}$ a vague tripled corrugation is visible in nearly all of the chains, with significantly enhanced intensity near defects. In the middle of longer chain segments the bulk period of $3.8 \AA$ is still visible through the superimposed (vague) tripled periodicity. At $110 \mathrm{~K}$, we observe a doubled periodicity in the bulk of most chains, but, again near defects, a tripled periodicity decaying into the chains is observed. From these data at higher temperatures, it is evident that the CDW present at $40 \mathrm{~K}$ nucleates from the defects and spreads along the chains with decreasing temperature. Even at RT, it is still possible to discern charge density oscillations emanating from the defects. These oscillations have been attributed to

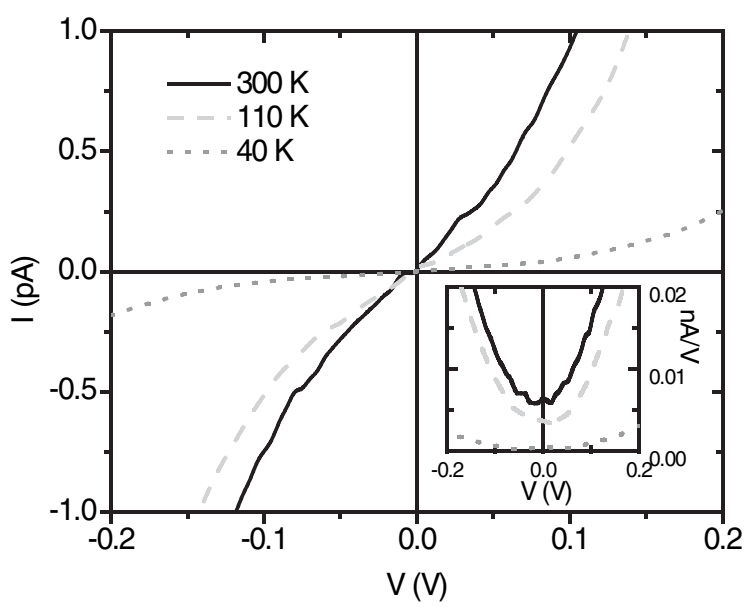

FIG. 3. Area averaged STS curves measured at indicated temperatures. Inset: numerical derivatives of the STS curves. 


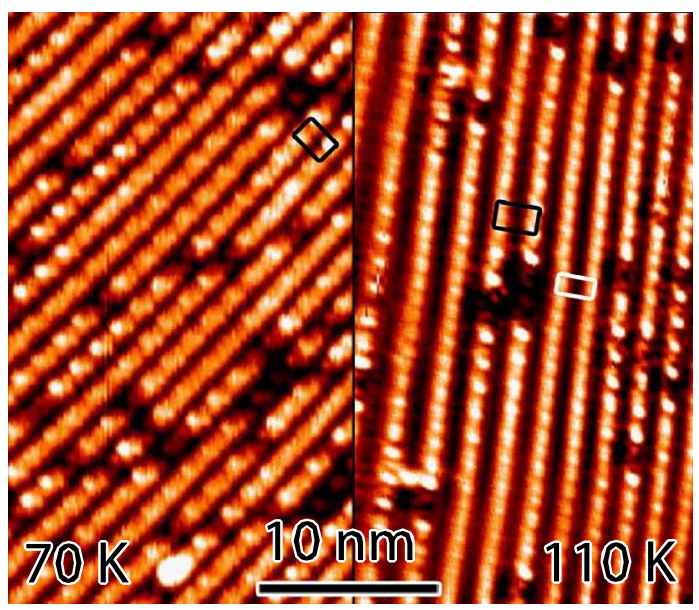

FIG. 4 (color online). Empty state (1 V, $50 \mathrm{pA})$ STM images at 70 and $110 \mathrm{~K} . \times 3$ and $\times 2$ unit cells are indicated in black and white open rectangles, respectively.

zero-dimensional end state effects [10]. Alternatively, the change in the apparent height of the chain adjacent to a defect, i.e., a depression over a distance of $\sim 1.5 a$ next to the defect followed by a brighter segment [see Fig. 1(a), inset], and the nonmetallic character of the chain ends established in Ref. [10] are fully consistent with the interpretation of a CDW precursor, similar to that observed in $\mathrm{Sn} / \mathrm{Ge}(111)$ [9].

As mentioned above, the band structure of this surface measured by ARPES contains three metallic bands [7]: two bands have a Fermi wave vector near half filling; the third band contains $0.27 \times 2=0.54$ electrons adding up to a total filling of $4 / 3$. None of the bands cross the Fermi energy exactly at a wave vector $2 \pi /(n \times a)$. Nevertheless, we observe three commensurate periodicities evolving as a function of temperature: at $110 \mathrm{~K}$ a clear $\times 2$ period is detected in the chains; at $40 \mathrm{~K}$ a $\times 2$ period is observed between the chains accompanied by a $\times 3$ period in the chains. Tentatively, we assign the three observed periods to electronic instabilities in the three metallic bands of the high-symmetry phase, which would locate the orbitals of the 0.51 and 0.27 filled bands on top of the zigzag chains and the orbitals of the 0.56 filled band in between the chains. At $110 \mathrm{~K}$, the doubled periodicity along the chains originates from a CDW transition in the 0.51 filled band; the other two bands remain metallic. STS measurements support this scenario, still showing metallic behavior at $110 \mathrm{~K}$ but with reduced slope at zero bias, indicating a reduced density of states (DOS) at the Fermi energy; see Fig. 3. ARPES at temperatures $<100 \mathrm{~K}$ show weak backfolding of the 0.27 filled band at the $\times 2$ zone boundary [7], also indicating a doubled periodicity in this band. The $\times 3$ CDW at lower temperatures can then be rationalized by a small charge transfer of 0.06 electron from the 0.56 filled band to the 0.27 filled band, filling the latter to $1 / 3$ [13]. The possible CDW precursor near the chain ends at RT can also be explained by this charge transfer: at $\mathrm{RT}$ a $\times 2$ period exists locally in between the chains near defects that are located on the chains [10]. This strongly suggests that already at RT the 0.56 filled band, located in the valleys between the chains, is doping the chains near the defects to $1 / 3$ filling. With this charge transfer the total band filling of the surface then remains constant at $4 / 3$. Note that this evolution in band structure might also explain the transformation of the features in the empty state STM image: from a zigzag structure at RT to a ladder structure at $110 \mathrm{~K}$ and below.

Surprisingly, the competition between periodicities inside the chains is eventually won by a $\times 3 \mathrm{CDW}$ at the lowest temperature studied. Longer wavelength periods are progressively more difficult to fit into chains with randomly placed fixed defects. Furthermore, a $1 / 3$ filled parabolic band has a higher DOS at the Fermi energy as compared to a $1 / 2$ filled band, which according to a simple BCS theory argument should result in a higher $T_{c}$ for the $1 / 3$ filled band. The magnitude of the interchain coupling for the different bands, though being fairly low as compared to other (bulk) 1D compounds [7], might provide insight into this seeming contradiction. It is well known that finite interchain coupling reduces the transition temperature, and indeed ARPES measurements indicate a 5 times larger interchain coupling for the $1 / 3$ filled band than for both of the $1 / 2$ filled bands. This could explain why the $\times 3 \mathrm{CDW}$ sets in at a lower temperature than the period doubling CDW observed at $110 \mathrm{~K}$ [15].

Finite interchain coupling should be discernible in the STM images through definite phase relations between periodicities in adjacent chains, provided that the temperature is low enough. Careful analysis of the empty state STM images at $40 \mathrm{~K}$ indeed reveals small domains of up to three or four chains width, showing a constant phase relation, but no long-range order is detected. However, as can be observed in Fig. 2(a) (arrow) even at $40 \mathrm{~K}$ there are chains which do not exhibit a fully developed CDW; the triple periodicity is not long-range ordered, despite the fact that a fully developed triple period would fit the length of this chain segment. This indicates that the system is affected by a significant amount of interchain coupling. This is further illustrated in Fig. 5(a). Two fairly long chains $(>25 \mathrm{~nm})$ are visible. Starting from the top, both chains lack clear corrugation, but both chains develop definite updown corrugation towards the bottom. The white bars illustrate a phase slip of $2 \pi / 3$ in the right chain, immediately followed by two similar phase slips in the left chain. These phase slips cannot be explained by a $\times 3 \mathrm{CDW}$ mismatch in a finite chain segment because both chains show a region of small corrugation at the top (and beyond, not shown) with an ill-defined phase so the CDW is not phase locked by defects. Therefore the origin must be related to interchain coupling; apparently, a phase slip in one chain can induce phase slips in neighboring chains via 


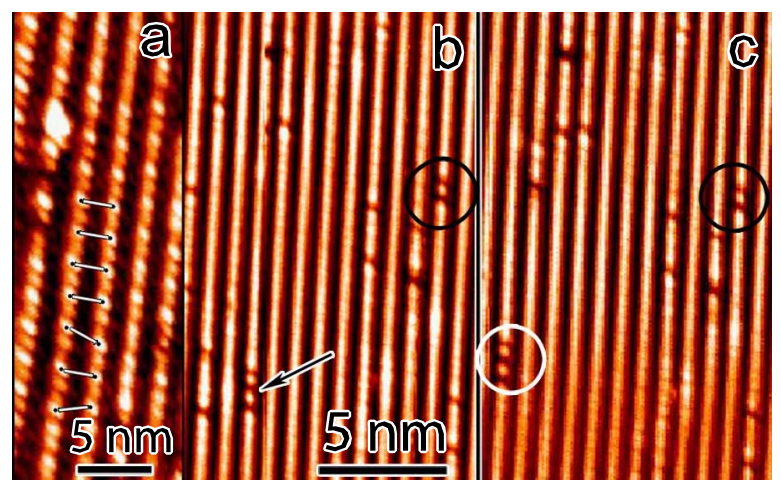

FIG. 5 (color online). STM images at $40 \mathrm{~K}$. (a) Empty state $(1 \mathrm{~V}, 100 \mathrm{pA})$ image showing phase slips in the $\times 3 \mathrm{CDW}$. (b),(c) Filled state $(-1 \mathrm{~V}, 50 \mathrm{pA})$ images showing relocation of defects. Black circles, markers; arrow, old defect position; white circle, new defect position.

interchain coupling. Interestingly, a phase slip (phase soliton) in a $1 / 3$ filled band CDW carries a (spin, charge) of $(1 / 2, \pm e / 3)$ or $(0, \pm 2 e / 3)$ [16].

Fractionally charged phase slips have been studied mostly theoretically. Only in polyacetylene, a phase slip with a fractional charge of $e / 2$ has been observed by conductivity and NMR experiments [17]. To the best of our knowledge, fractionally charged phase slips in CDWs away from half filling have never been observed directly by imaging. Their presence opens up the possibility to study and manipulate fractional charges at the atomic scale. They can possibly be manipulated by deliberately creating defects in the chains so as to generate a misfit for the $\times 3$ CDW. In our STM experiments, at $40 \mathrm{~K}$ defects sometimes jump to different locations during imaging. For example, Figs. 5(b) and 5(c) show two sequential $(\Delta t=180 \mathrm{~s})$ filled state images from the same area. The white circle and the arrow show a group of three defects, which has moved two chains to the left. This behavior suggests that these defects consist of Si adatoms [7,8], which have been relocated by tip induced migration.

In conclusion, we have presented an STM and STS study of a chain structure with fractional band fillings. Competing periodicities are observed as a function of temperature resulting in a defect mediated $\mathrm{CDW}$ at $40 \mathrm{~K}$. The results can be mapped onto the band structure of the highsymmetry phase. The presence and mobility of the chain dislocations in the CDW state indicate the possibility of studying and possibly manipulating fractionally charged solitons with an STM tip. The availability of other vicinal $\mathrm{Si}$-Au-chain structures with tunable interchain coupling [7] would provide a promising playground for 1D physics, accessible in real space.

We thank Professor Franz Himpsel for providing the $\mathrm{Si}(553)$ wafer. This work is sponsored in part by the NSF under Contract No. DMR-0244570, the Stichting voor Fundamenteel Onderzoek der Materie, and the Royal Netherlands Academy of Arts and Sciences. We thank T. M. Klapwijk for his stimulating support. Oak Ridge National Laboratory is managed by UT-Battelle, LLC, for the U.S. Department of Energy under Contract No. DE-AC-05-00OR22725.

Note added.-After the submission of our manuscript, we became aware of the Letter by Ahn et al. [18]. Their observations are consistent with ours.

[1] N. D. Mermin and H. Wagner, Phys. Rev. Lett. 17, 1133 (1966); N. D. Mermin, Phys. Rev. 176, 250 (1968).

[2] M. J. Rice and S. Strässler, Solid State Commun. 13, 1389 (1973); E. Pytte, Phys. Rev. B 10, 2039 (1974).

[3] M. Lee, E. A. Kim, J. S. Lim, and M. Y. Choi, Phys. Rev. B 69, 115117 (2004); N. Shannon and R. Joynt, J. Phys. Condens. Matter 8, 10493 (1996).

[4] G. Grüner and A. Zettl, Phys. Rep. 119, 117 (1985).

[5] H. W. Yeom et al., Phys. Rev. B 72, 035323 (2005).

[6] H. W. Yeom, K. Horikoshi, H. M. Zhang, K. Ono, and R. I. G. Uhrberg, Phys. Rev. B 65, 241307(R) (2002); S. J. Park, H. W. Yeom, S. H. Min, D. H. Park, and I. W. Lyo, Phys. Rev. Lett. 93, 106402 (2004); G. Lee, J. Guo, and E. W. Plummer, Phys. Rev. Lett. 95, 116103 (2005); H. Morikawa, I. Matsuda, and S. Hasegawa, Phys. Rev. B 70, 085412 (2004); J. Guo, G. Lee, and E. W. Plummer, Phys. Rev. Lett. 95, 046102 (2005).

[7] J. N. Crain et al., Phys. Rev. Lett. 90, 176805 (2003); Phys. Rev. B 69, 125401 (2004).

[8] S. Riikonen and D. Sanchez-Portal, Nanotechnology 16, S218 (2005).

[9] H. H. Weitering et al., Science 285, 2107 (1999).

[10] J. N. Crain and D. T. Pierce, Science 307, 703 (2005).

[11] S. C. Erwin and H. H. Weitering, Phys. Rev. Lett. 81, 2296 (1998).

[12] J. Avila et al., Phys. Rev. Lett. 82, 442 (1999).

[13] According to Ref. [14], a commensurate CDW in a system with incommensurate Fermi wave vectors can be obtained by locking the wavelength of the CDW to the lattice so as to be commensurate. However, then it would be expected that the 0.27 filled band would lock to a $\times 4$ period corresponding to a filling of 0.25 .

[14] J.R. Schrieffer, Nobel Symposium 24 (Academic, New York, 1973), p. 142.

[15] An alternative explanation is that the relatively large charge transfer into the 0.27 filled band induces significantly more lattice strain, thereby lowering its $T_{c}$ as compared to the $\times 2 \mathrm{CDW}$.

[16] W.P. Su and J. R. Schrieffer, Phys. Rev. Lett. 46, 738 (1981); C. Kuhn, J. Phys. Condens. Matter 7, 6221 (1995).

[17] W. P. Su, J. R. Schrieffer, and A. J. Heeger, Phys. Rev. B 22, 2099 (1980).

[18] J. R. Ahn et al., Phys. Rev. Lett. 95, 196402 (2005). 